\title{
Mucoadhesive Electrospun Patch Delivery of Lidocaine to the Oral Mucosa and Investigation of Spatial Distribution in a Tissue Using MALDI-Mass Spectrometry Imaging
}

Clitherow, Katharina H.; Murdoch, Craig; Spain, Sebastian Guy; Handler, Anna Mette; Colley, Helen E.; Stie, Mai Bay; Nielsen, Hanne Morck; Janfelt, Christian; Hatton, Paul V.; Jacobsen, Jette

Published in:

Molecular Pharmaceutics

DOI:

10.1021/acs.molpharmaceut.9b00535

Publication date:

2019

Document version

Publisher's PDF, also known as Version of record

Document license:

CC BY

Citation for published version (APA):

Clitherow, K. H., Murdoch, C., Spain, S. G., Handler, A. M., Colley, H. E., Stie, M. B., Nielsen, H. M., Janfelt, C., Hatton, P. V., \& Jacobsen, J. (2019). Mucoadhesive Electrospun Patch Delivery of Lidocaine to the Oral Mucosa and Investigation of Spatial Distribution in a Tissue Using MALDI-Mass Spectrometry Imaging. Molecular Pharmaceutics, 16(9), 3948-3956. https://doi.org/10.1021/acs.molpharmaceut.9b00535 


\title{
Mucoadhesive Electrospun Patch Delivery of Lidocaine to the Oral Mucosa and Investigation of Spatial Distribution in a Tissue Using MALDI-Mass Spectrometry Imaging
}

\author{
Katharina H. Clitherow, ${ }^{\dagger}$ Craig Murdoch, ${ }^{\dagger}$ Sebastian Guy Spain, ${ }^{\ddagger}$ Anna Mette Handler, ${ }^{\S}$
}

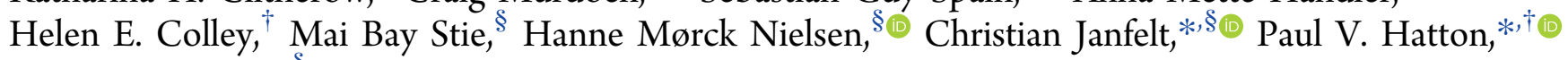
and Jette Jacobsen ${ }^{\S}$

${ }^{\dagger}$ School of Clinical Dentistry, University of Sheffield, 19 Claremont Crescent, Sheffield S10 2TA, U.K.

${ }^{\ddagger}$ Department of Chemistry, University of Sheffield, Brook Hill, Sheffield S3 7HF, U.K.

${ }^{\S}$ Department of Pharmacy, University of Copenhagen, 2 Universitetsparken, Copenhagen DK-2100, Denmark

\section{Supporting Information}

\begin{abstract}
Many oral mucosal conditions cause considerable and prolonged pain that to date has been difficult to alleviate via topical delivery, and the use of injection causes many patients dental anxiety and needle-prick pain. Therefore, developing a noninjectable drug delivery system as an alternative administration procedure may vastly improve the health and wellbeing of these patients. Recent advances in the development of mucoadhesive electrospun patches for the direct delivery of therapeutics to the

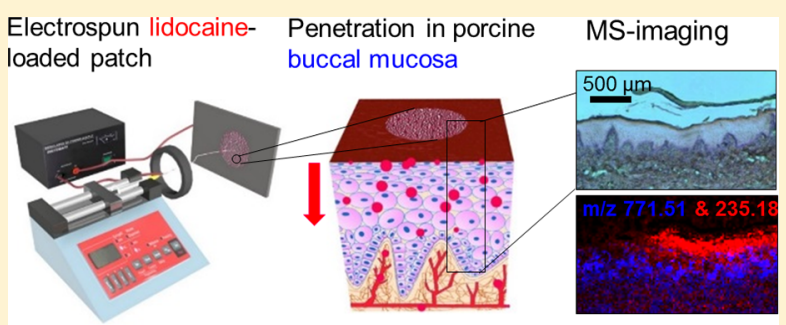
oral mucosa offer a potential solution, but as yet, the release of local anesthetics from this system and their uptake by oral tissue have not been demonstrated. Here, we demonstrate the fabrication of lidocaine-loaded electrospun fiber patches, drug release, and subsequent uptake and permeation through the porcine buccal mucosa. Lidocaine $\mathrm{HCl}$ and lidocaine base were incorporated into the electrospun patches to evaluate the difference in drug permeation for the two drug compositions. Lidocaine released from the lidocaine $\mathrm{HCl}$-containing electrospun patches was significantly quicker than from the lidocaine base patches, with double the amount of drug released from the lidocaine $\mathrm{HCl}$ patches in the first $15 \mathrm{~min}(0.16 \pm 0.04 \mathrm{mg})$ compared to that from the lidocaine base patches $(0.07 \pm 0.01 \mathrm{mg})$. The permeation of lidocaine from the lidocaine $\mathrm{HCl}$ electrospun patches through ex vivo porcine buccal mucosa was also detected in $15 \mathrm{~min}$, whereas permeation of lidocaine from the lidocaine base patch was not detected. Matrix-assisted laser desorption ionization-mass spectrometry imaging was used to investigate localization of lidocaine within the oral tissue. Lidocaine in the solution as well as from the mucoadhesive patch penetrated into the buccal mucosal tissue in a time-dependent manner and was detectable in the lamina propria after only $15 \mathrm{~min}$. Moreover, the lidocaine released from lidocaine $\mathrm{HCl}$ electrospun patches retained biological activity, inhibiting veratridine-mediated opening of voltage-gated sodium channels in SH-SY5Y neuroblastoma cells. These data suggest that a mucoadhesive electrospun patch may be used as a vehicle for rapid uptake and sustained anesthetic drug delivery to treat or prevent oral pain.
\end{abstract}

KEYWORDS: electrospinning, lidocaine, oral mucosa, ex vivo tissue permeation, MALDI-mass spectrometry imaging, drug distribution in tissue

\section{INTRODUCTION}

Control of pain is a major unmet clinical need in a range of oral conditions including mucositis, ulceration, and trauma. ${ }^{1,2}$ The effects of pain may be so severe as to affect eating, drinking, speech, or sleep. ${ }^{3}$ Pain is also associated with a number of dental procedures and is most commonly alleviated by the injection of a local anesthetic. Unfortunately, the use of injection is a major factor in dental anxiety and nonattendance at the clinic. ${ }^{4} \mathrm{~A}$ wide range of technologies to alleviate localized pain in the oral cavity have been investigated, including tablets, films, gels, and mouth washes. ${ }^{5-10}$ These are chiefly based on topical delivery of drugs, but all current approaches have limitations related to the very challenging conditions found within the oral cavity, where salivary flow and tissue movement contribute to a hostile mechanical and chemical environment.

We have recently fabricated an electrospun patch with mucoadhesive properties with the potential to deliver drugs to oral lesions for a sustained period. ${ }^{11}$ The patch displays good adhesion to oral tissues and is well tolerated with no significant cytotoxicity or irritation in both a porcine model and healthy

Received: May 16, 2019

Revised: July 26, 2019

Accepted: July 30, 2019

Published: July 30, 2019 
volunteers. $^{12}$ The delivery of clobetasol-17-propionate was demonstrated in a tissue-engineered oral mucosal model and in ex vivo porcine mucosa. ${ }^{12}$ Electrospinning can create relatively thin-fiber (typically $100 \mathrm{~nm}$ to $50 \mu \mathrm{m}$ fiber diameter) patches, where the polymer and solvent selection, as well as the electrospinning conditions, may alter the mechanical properties of the mat and its behavior in the oral cavity. ${ }^{13}$ These electrospun patches have high mucoadhesive properties due to the high surface area to volume ratio and to the incorporation of a hygroscopic polymer that rapidly adsorbs saliva at the site of application leading to rapid and prolonged drug release. ${ }^{11,14}$ This innovative medical device has considerable potential for the site-specific delivery of pain-relieving drugs such as nonsteroidal anti-inflammatories or local anesthetics. ${ }^{12,15}$

Lidocaine $\mathrm{HCl}$ is a relatively safe and effective anesthetic and most commonly used in local oromucosal pain relief. ${ }^{16} \mathrm{~A}$ number of ex vivo studies evaluating the transbuccal permeation of lidocaine, applied as either a solution or gel, have been reported. ${ }^{17,18}$ However, electrospun patches have not been subject to a detailed investigation of transbuccal delivery of lidocaine. Porcine mucosa is frequently used in transbuccal permeation experiments since it is physiologically similar to that of humans, and is, therefore, considered the gold standard for modeling oral drug delivery. ${ }^{19}$ Ex vivo permeation studies provide quantitative data; however, to visualize the spatial distribution of drugs in the tissue, mass spectrometry imaging (MSI) may be used. MSI allows for a multitude of compounds to be detected including the drug, metabolites, excipients, and endogenous compounds, such as lipids, which may serve as tissue biomarkers. There are a number of different MSI techniques, with matrix-assisted laser desorption ionization (MALDI) MSI being one of the most commonly used. ${ }^{20}$ The method requires deposition of a matrix on the tissue sample to aid the ionization of analytes when targeted by the laser. The process is relatively nondestructive, and, therefore, the tissue sample can be stained using hematoxylin and eosin (H\&E) for histological evaluation after the process. We have previously used MALDI-MSI to examine permeation of nicotine across porcine buccal mucosa, ${ }^{21,22}$ and a similar experimental setup was used in this study to determine the time-dependent permeation of lidocaine.

Herein, we demonstrate for the first time the targeted permeation of lidocaine into ex vivo porcine buccal mucosa released from mucoadhesive electrospun patches to be used as a local oromucosal drug delivery vehicle. Both lidocaine $\mathrm{HCl}$ and lidocaine base were successfully incorporated into the electrospun patches, for which the release, ex vivo permeation, and bioactivity were measured. Our findings show a greater release of lidocaine $\mathrm{HCl}$ from the electrospun patches compared to lidocaine base, and only lidocaine permeation from the lidocaine $\mathrm{HCl}$ patches was detected ex vivo. Additionally, using MALDI-MSI, the localization and timedependent penetration of the drug within the tissue were established. This study shows that lidocaine-loaded electrospun patches deliver functionally active drug directly to the buccal mucosa in a therapeutically useful timeframe. This drug delivery device has the potential of improving the control of localized oral pain that is currently a major unmet clinical problem.

\section{METHODS}

Materials. Poly(vinylpyrrolidone) (PVP) $\left(M_{\mathrm{W}} 2000 \mathrm{kDa}\right)$ and Eudragit RS100 $\left(M_{\mathrm{W}} 30 \mathrm{kDa}\right)$ were kindly donated by
BASF (U.K.) and Evonik Industries AG (Germany), respectively. Poly(caprolactone) (PCL) $\left(M_{\mathrm{W}} 80 \mathrm{kDa}\right)$, poly(ethylene oxide) (PEO) $\left(M_{\mathrm{W}} 400 \mathrm{kDa}\right)$, trifluoroacetic acid (TFA, 99\%), 2,5-dihydroxybenzanoic acid (DHB, 98\%), carboxymethylcellulose (CMC), potassium dihydrogen phosphate, disodium hydrogen phosphate, sodium hydroxide, alcoholic eosin Y solution, hematoxylin solution Mayer's, fetal calf serum, nonessential amino acids, penicillin, streptomycin, ethylenediaminetetraacetic acid (EDTA), dimethylsulfoxide (DMSO) were purchased from Sigma-Aldrich (U.K. \& DK). Lidocaine $\mathrm{HCl}\left(M_{\mathrm{W}} 270.8 \mathrm{Da}\right)$, lidocaine $\left(M_{\mathrm{W}}\right.$ $234.3 \mathrm{Da})$, and veratridine were purchased from Abcam (U.K.). Solvents such as ethanol ( $\geq 99.8 \%)$, dichloromethane (DCM, $\geq 99 \%$ ), dimethylformamide (DMF, 99.98\%), and acetonitrile (ACN, $\geq 99.9 \%$ ), media such as DMEM/Ham's F12 with L-glutamine and Fluo-4 Direct assay kit (Invitrogen) were purchased from Fisher Scientific (U.K.). Human neuroblastoma cell line SH-SY5Y was purchased from LGC Standards (U.K.). Methanol ( $\geq 99.8 \%$ ) and sodium chloride were obtained from Th. Geyer GmbH and Co. KG (Germany). Ultrapure water was collected from the water system SG Ultra Clear 2002 from Evoqua Water Technologies LLC. Isotonic phosphate-buffered saline (PBS) was prepared by dissolving $2.38 \mathrm{~g}$ of disodium hydrogen phosphate with 0.19 $\mathrm{g}$ of potassium dihydrogen phosphate and $8 \mathrm{~g}$ of sodium chloride in $1 \mathrm{~L}$ of ultrapure water and adjusted with hydrochloric acid and sodium chloride to achieve a $\mathrm{pH}$ of 7.4 and an osmotic pressure of $290 \mathrm{mOsm} \mathrm{kg}$.

Electrospun Patch Manufacture. Electrospun mucoadhesive patches were produced as described by Santocildes et al. ${ }^{11}$ with modifications. Briefly, the electrospun dope consisted of $10 \%(\mathrm{w} / \mathrm{w})$ PVP, $12.5 \%(\mathrm{w} / \mathrm{w})$ Eudragit RS100, and $10 \%$ $(\mathrm{w} / \mathrm{w})$ PEO $(400 \mathrm{kDa})$ and amounts of lidocaine base or lidocaine $\mathrm{HCl}$ theoretically resulting in a $3 \%(\mathrm{w} / \mathrm{w})$ drug loading in the electrospun patch (i.e., $0.1 \mathrm{~g}$ of either lidocaine base or lidocaine $\mathrm{HCl}$ added to $0.326 \mathrm{~g}$ of polymers in the dope solution). In the case of lidocaine $\mathrm{HCl}$, the $3 \%(\mathrm{w} / \mathrm{w})$ loading includes the salt content. Polymers and drug were dissolved in $97 \%(\mathrm{w} / \mathrm{w})$ ethanol prepared in deionized water, and solutions mixed at room temperature using a magnetic stirrer for $24 \mathrm{~h}$ or until they had dissolved. The electrospinning rig was configured with the following parameters: $19 \mathrm{kV}$ voltage, $14 \mathrm{~cm}$ tip-to-collector distance, and $1.5 \mathrm{~mL} \mathrm{~h}^{-1}$ flow rate. Additionally, $10 \%(\mathrm{w} / \mathrm{w})$ PCL dissolved in DCM/DMF in a ratio of 93:7 (w/w) was electrospun using the same conditions and used as a backing layer. ${ }^{11}$ The dual-layer patch was formed by melting the PCL backing layer onto the drugcontaining mucoadhesive layer by placing the layers between two glass slides followed by incubation at $70{ }^{\circ} \mathrm{C}$ for $5 \mathrm{~min}$.

Characterization of Electrospun Patches. The morphology of the different electrospun patches was analyzed using scanning electron microscopy (SEM). The samples were gold-sputtered and imaged using Tescan Vega3 LMU SEM (U.K.) with a high accelerating voltage of $15 \mathrm{kV}$. The fiber diameters were subsequently measured from the SEM images for the non-drug-loaded and drug-loaded patches $(n=3$ patches for each condition, $\sim 35$ fiber measurements per image) using the ImageJ software (National Institute of Health). ${ }^{23}$ Differential scanning calorimetry (DSC) was applied using a Pyris 1 calorimeter (PerkinElmer) to determine the temperature transitions of the drug- and non-drugcontaining electrospun patches. Electrospun patches (5-9 $\mathrm{mg})$, lidocaine $\mathrm{HCl}$ or lidocaine base powders $(\sim 3 \mathrm{mg})$ were 
weighed and placed in $50 \mu \mathrm{L}$ aluminum pans, and aluminum lids crimped on top. Patches or powders were heated from 25 to $200{ }^{\circ} \mathrm{C}$ at $10{ }^{\circ} \mathrm{C} \mathrm{min}^{-1}$. The $\mathrm{pH}$ was measured after fully dissolving the different electrospun patches $(n=3$ patches for each condition, $110 \pm 4 \mathrm{mg}$; mean \pm standard deviation (SD)) in $10 \mathrm{~mL}$ of deionized water using a FiveGo $\mathrm{pH}$ meter (Mettler Toledo, Switzerland) at $20.5{ }^{\circ} \mathrm{C}$.

Quantification of Lidocaine. Reverse phase high performance liquid chromatography (RP-HPLC) with UV detection was performed to detect the presence of lidocaine using a XBridge BEH-C18 column $(4.6 \mathrm{~mm} \times 250 \mathrm{~mm}$; $130 \AA$ pore size) (Waters, U.K.) and a mobile phase composed of $\mathrm{ACN} /$ water $(1: 1, \mathrm{v} / \mathrm{v})$ containing $0.1 \%(\mathrm{v} / \mathrm{v}) 7.5 \mathrm{M}$ ammonia, with a flow rate of $1 \mathrm{~mL} \mathrm{~min}^{-1}$, wavelength $262 \mathrm{~nm}$, a $20 \mu \mathrm{L}$ injection volume, and at room temperature. The retention time of lidocaine was $10.1 \mathrm{~min}$, and the method was validated in terms of linearity and precision using lidocaine $\mathrm{HCl}$ in $\mathrm{PBS}$ standards of known concentrations. Good linearity $\left(R^{2}=1.00\right)$ was determined with repeated injections of lidocaine $\mathrm{HCl}$ in PBS ranging from 39 to $1250 \mu \mathrm{M}$, where previous lower-range concentration injections determined $1.8 \mu \mathrm{M}$ as the limit of quantitation (10 times the background reading created by PBS in the lidocaine peak region).

Dosage Uniformity of Lidocaine in the Electrospun Patches. Electrospun patches containing lidocaine $\mathrm{HCl}$ or lidocaine base were weighed, dissolved in $1 \mathrm{~mL}$ of $99.8 \%$ ethanol with stirring, and analyzed by UV RP-HPLC. Electrospun samples $(n=3)$ were taken from three separately spun mats to determine batch variation, where lidocaine $\mathrm{HCl}$ patches weighed $6.2 \pm 1.2 \mathrm{mg}$ and lidocaine base patches weighed $9.3 \pm 3.3 \mathrm{mg}$.

Release of Lidocaine from the Electrospun Patches in the Buffer. Electrospun patches $(\varnothing 10 \mathrm{~mm})$ containing lidocaine $\mathrm{HCl}$ or lidocaine base were weighed and placed in a 12 -well plate containing $2 \mathrm{~mL}$ of PBS. Samples of $200 \mu \mathrm{L}$ were withdrawn every $5 \mathrm{~min}$ for $1 \mathrm{~h}$, with the solution being replaced with a fresh buffer each time. The amount of lidocaine released was quantified using the RP-HPLC method previously described.

Tissue Preparation. Ex vivo porcine buccal mucosa was obtained from healthy experimental control pigs (approximately $30 \mathrm{~kg}$ of Danish Landrace/Yorkshire $\times$ Durox (D-LY)) (Department of Experimental Medicine, University of Copenhagen) and stored at $-80{ }^{\circ} \mathrm{C}$ in cryomedia $(40 \%$ (w/ v) glycerol, $20 \%(\mathrm{w} / \mathrm{v})$ sucrose in PBS at $\mathrm{pH} 7.4$ and 290 mOsm kg-1). ${ }^{24}$ The mucosa was thawed and washed by removal from the cryomedia into $4{ }^{\circ} \mathrm{C}$ PBS positioned on a 4 ${ }^{\circ} \mathrm{C}$ rotating plate and transferred to fresh PBS four times with 10 min incubations. The submucosa was trimmed to a thickness of $1011 \pm 133 \mu \mathrm{m}$ (mean $\pm \mathrm{SD}, n=10)$ and mounted onto an Ussing slider (P2413, Physiological Instruments Inc.) with an exposed area of $0.71 \mathrm{~cm}^{2}$.

Buccal Mucosa Permeability Study. Permeability studies were performed using modular EM-CSY-8 modified Ussing chambers (Physiologic Instruments Inc.). Receptor chambers were filled with $2 \mathrm{~mL}$ of PBS ( $\mathrm{pH}$ 7.4) for all experiments. Donor chambers either contained $2 \mathrm{~mL}$ PBS with $3 \%(\mathrm{w} / \mathrm{v})$ lidocaine $\mathrm{HCl}(\mathrm{pH}$ 6.8) or were left empty for lidocainecontaining electrospun patch experiments. Patches were punched from the dual-layer electrospun mats $(\varnothing 10 \mathrm{~mm})$, and the drug-containing side of the patch was placed on the buccal epithelium and held in place with Parafilm $M$ to assure direct contact of the patch with the tissue for the duration of the experiment (Figure 1). The dual-layer patches containing lidocaine $\mathrm{HCl}$ weighed $15.6 \pm 2.3 \mathrm{mg}$ (mean $\pm \mathrm{SD}, n=10)$,

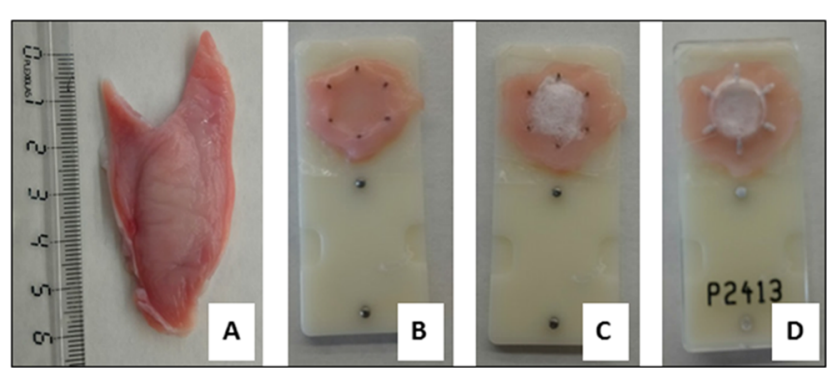

Figure 1. Preparation of porcine buccal mucosa for the lidocaine diffusion study. (A) Defrosted porcine buccal mucosa (ruler only associated to this image); (B) buccal mucosa cut to size and stretched across the Ussing slider with the epithelium facing up; (C) electrospun patch on the epithelium surface; (D) Parafilm M stretched across the sample to hold the electrospun patch in place and sandwiched together with the second part of the slider.

where the backing membrane PCL layer weighed $3.2 \pm 0.14$ mg (mean $\pm \mathrm{SD}, n=3)$. Sink conditions were maintained in the receptor chambers. A slight temperature increase over the $5 \mathrm{~h}$ period was observed $\left(\sim 37.0\right.$ to $\left.\sim 38.4{ }^{\circ} \mathrm{C}\right)$.

Aliquots of $200 \mu \mathrm{L}$ were taken from the receptor chamber every 15-30 min over a $5 \mathrm{~h}$ period and were replaced with fresh PBS at each time point. The lidocaine concentration of each sample was determined using UV-HPLC. The accumulated permeated amount $\left(J_{S S}, \mu \mathrm{g} \min ^{-1} \mathrm{~cm}^{-2}\right)$ was plotted against time $(t, \min )$, and the steady-state flux $(\mathrm{d} Q / \mathrm{d} t, \mu \mathrm{g}$ $\left.\min ^{-1}\right)$, where $Q(\mu \mathrm{g})$ is the drug concentration, was determined by taking the linear part of the slope between 180 and $300 \mathrm{~min}$ using the eq 1 for steady-state flux $\left(J_{S S}\right)$, where $A$ is the diffusion area $\left(0.71 \mathrm{~cm}^{2}\right)$

$$
J_{\text {SS }}=\frac{\mathrm{d} Q}{\mathrm{~d} t} \times \frac{1}{A}
$$

For lidocaine $\mathrm{HCl}$ in $\mathrm{PBS}$, the apparent permeability $\left(P_{\text {app }}, \mathrm{cm}\right.$ $\min ^{-1}$ ) was calculated using eq 2

$$
P_{\text {app }}=\frac{J_{\text {SS }}}{C_{\text {donor }}}
$$

where the donor concentration was $C_{\text {donor }}\left(\mu \mathrm{g} \mathrm{L}^{-1}\right)$.

Spatial Location of Lidocaine in Buccal Mucosal Using MALDI-MSI. Buccal mucosa was thawed, cut to a thickness of approximately $5 \mathrm{~mm}$ and mounted onto Ussing sliders. Three different experimental conditions were imposed on mounted tissues: (1) $70 \mu \mathrm{L}$ of $0.3 \%(\mathrm{w} / \mathrm{v})$ lidocaine $\mathrm{HCl}$ in PBS directly pipetted onto the epithelium; (2) an electrospun patch containing lidocaine $\mathrm{HCl}$ applied to the epithelium and held in place using Parafilm M; (3) an electrospun patch containing lidocaine base applied to the epithelium and held in place using Parafilm M. Three tissues for each condition were prepared, and the sliders were placed in a high humidity chamber filled at the bottom with PBS to cover the connective tissue side and incubated at $37.4{ }^{\circ} \mathrm{C}$. Tissues were removed from the chamber after $15 \mathrm{~min}, 1$ and $3 \mathrm{~h}$. Samples were immediately embedded in $5 \%(\mathrm{w} / \mathrm{v}) \mathrm{CMC}$ and stored at -80 ${ }^{\circ} \mathrm{C}$. Frozen tissue samples were mounted in a Leica CM $3050 \mathrm{~S}$ Cryostat (Leica Microsystems A/S, Germany), cut vertically at $-20{ }^{\circ} \mathrm{C}$ into $30 \mu \mathrm{m}$ thick cross-sections, thaw-mounted onto glass microscopic slides that were stored at $-80{ }^{\circ} \mathrm{C}$. Sections 

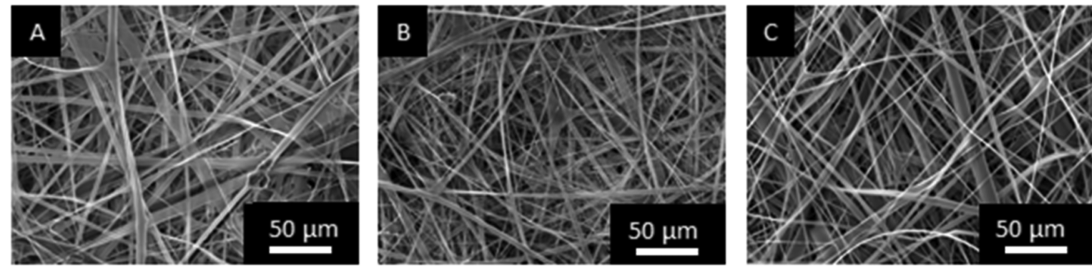

Figure 2. Representative SEM images of electrospun patches from $n=3$ batches where (A) is the drug-free patch, (B) patch loaded with $3 \%$ (w/w) lidocaine $\mathrm{HCl}$, and $(\mathrm{C})$ patch loaded with $3 \%(\mathrm{w} / \mathrm{w})$ lidocaine base.

were removed from $-80{ }^{\circ} \mathrm{C}$ and thawed using a vacuum desiccator for 5-10 min, then $300 \mu \mathrm{L}$ of the matrix solution (30 $\mathrm{mg} \mathrm{mL}^{-1}$ of 2,5-dihydroxybenzoic acid (DHB) in methanol/water $(1: 1, \mathrm{v} / \mathrm{v})$ with $1 \%(\mathrm{v} / \mathrm{v})$ TFA) was sprayed onto the sample at a flow rate of $30 \mu \mathrm{L} \mathrm{min}{ }^{-1}$ with a nebulized gas pressure of 2 bar while the sample was rotating at $550 \mathrm{rpm}$, approximately $10 \mathrm{~cm}$ from the syringe tip. An AP-SMALDI10 Ion source (TransMIT Gesellschaft für Technologietransfer $\mathrm{GmbH}$, Germany) mounted on a QExactive Orbitrap mass spectrometer (Thermo Scientific GmbH, Germany) was used for MALDI imaging. Imaging was performed in the positive ion mode using a scan range of $\mathrm{m} / z \quad 200-800$ and mass resolving power of $140000 @ m / z 200$. A peak from the DHB matrix at $m / z$-value of 295.02131 was used as a lock mass, thereby ensuring a mass accuracy of at least $2 \mathrm{ppm}$ throughout the entire image. The images were acquired with a pixel size of $20 \mu \mathrm{m}$, and no oversampling took place (no overlapping between adjacent ablation craters was confirmed by reflected light microscopy of the samples subsequently to the MALDI imaging experiment). The raw data were converted to imzML files, ${ }^{25}$ and the MALDI images were generated in a MSiReader v. $0.09^{26}$ using a bin width of $0.002 \mathrm{Th}$.

Histological Staining. Post-MSI, the DHB matrix was removed by washing with ethanol and tissue sections stained with hematoxylin and eosin as previously described, ${ }^{27}$ then imaged using an Olympus BH-2 microscope (Olympus, Japan) equipped with an AxioCam ERc5s camera (Zeiss, Germany).

Lidocaine Functional Assay. The human neuroblastoma cell line SH-SY5Y was cultured in 1:1 (v/v) DMEM/Ham's F12, supplemented with $10 \%$ fetal calf serum, $2 \mathrm{mM} \mathrm{L-}$ glutamine, $1 \%$ nonessential amino acids, $100 \mathrm{IU} \mathrm{mL}^{-1}$ of penicillin, and $100 \mu \mathrm{g} \mathrm{mL}^{-1}$ of streptomycin and incubated at $37{ }^{\circ} \mathrm{C}, 5 \% \mathrm{CO}_{2}$ in a humidified environment. Prior to experiments, cells were loaded with the fluorescent calcium indicator, Fluo-4 Direct following the manufacturer's instructions. Briefly, cells were removed from tissue culture flasks using EDTA, centrifuged, and resuspended at $10^{6}$ cells/ $\mathrm{mL}$ in a Fluo-4 Direct calcium buffer for $30 \mathrm{~min}$ at $37^{\circ} \mathrm{C}$. To test lidocaine bioactivity, lidocaine $\mathrm{HCl}$-loaded or placebo electrospun patches (containing no lidocaine) were cut to $2 \times$ $2 \mathrm{~cm}^{2}$ and fully dissolved in $2 \mathrm{~mL}$ of PBS for $30 \mathrm{~min}$, filtersterilized, then the solution added to Fluo-4 Direct-loaded cells to give a final concentration of $0.5 \mathrm{mM}$ lidocaine or placebo control, and the cells incubated for a further $30 \mathrm{~min}$ at room temperature. To determine cell calcium responses, fluorescence was measured at $488 \mathrm{~nm}$ excitation and 530/30 nm emission using a FACSCalibur (BD Biosciences). For each sample, baseline fluorescence was measured for $40 \mathrm{~s}$, then veratridine or vehicle control DMSO was added, and the fluorescence response measured for approximately a further 160 s. Relative fluorescence units (RFU) were calculated by subtracting the baseline median fluorescence intensity from the maximal median fluorescence intensity following stimulation with veratridine.

\section{RESULTS}

Manufacture of the Electrospun Patches. Mucoadhesive electrospun patches containing lidocaine or lidocaine $\mathrm{HCl}$ were produced by modification of our published protocol. ${ }^{11}$ SEM was used to determine if the inclusion of lidocaine affected the structure or morphology of the patches (Figure 2). Patches of three different conditions were manufactured; patches containing lidocaine $\mathrm{HCl}$ or lidocaine base along with control patches without the drug. SEM images show that there was no significant difference in the fiber diameter or fiber structure between drug-containing and drug-free patches (Figure 2). All patches showed a similar range of fiber diameters with a random alignment, where the drug-free fiber diameters ranged from $2.28 \pm 1.35 \mu \mathrm{m}$, the lidocaine $\mathrm{HCl}$ loaded fibers from $1.98 \pm 1.50 \mu \mathrm{m}$, and the lidocaine baseloaded fibers from $2.42 \pm 2.09 \mu \mathrm{m}(\sim 100$ fibers in total measured from $n=3$ patches; mean \pm SD). As two different drug compositions are incorporated into the patches and drug release can be dependent on the $\mathrm{pH}$ microenvironment of a system, the $\mathrm{pH}$ of the patches dissolved in deionized water was measured. It was found that the $\mathrm{pH}$ of the non-drug-containing patches in water was $7.78 \pm 0.03$, the $\mathrm{pH}$ of lidocaine $\mathrm{HCl}$ containing patches in water was $7.25 \pm 0.04$, and the $\mathrm{pH}$ for the lidocaine base-containing patches in water was $8.26 \pm 0.13$ $(n=3$; mean $\pm \mathrm{SD})$.

Lidocaine Content of the Electrospun Patches. Lidocaine was added to the polymer dope to achieve a drug loading of $3 \%(\mathrm{w} / \mathrm{w})$. As low-molecular-weight species can be lost during spinning, ${ }^{28,29}$ the content of lidocaine in the patches was determined by HPLC. Some variations in the total drug content between patches were observed, with patches containing $2.4 \pm 0.5 \%(\mathrm{w} / \mathrm{w})$ lidocaine $\mathrm{HCl}$ or $2.5 \pm 0.2 \%(\mathrm{w} /$ w) lidocaine base $(n=3)$. Drug solubility for improved bioavailability is of great importance in drug delivery, and this may be improved by developing high-energy amorphous systems. ${ }^{30}$ In this case, the drug is encapsulated in polymer fibers and needs to be released prior to penetration into the tissue. DSC analysis showed that the respective active pharmaceutical ingredients are in an amorphous form within the electrospun fibers (Figure 3). That is, the data showed the absence of a peak at $81{ }^{\circ} \mathrm{C}$ in the lidocaine $\mathrm{HCl}$-containing patch, where lidocaine $\mathrm{HCl}$ powder has its melting peak, and the absence of a peak at $70{ }^{\circ} \mathrm{C}$, for the lidocaine basecontaining patch, the melting peak of the lidocaine base powder.

Release of Lidocaine from the Electrospun Patches into the Buffer. The mean accumulative release of lidocaine $\mathrm{HCl}$ and lidocaine base from four electrospun patches for each condition over $1 \mathrm{~h}$ is shown in Figure 4, with respective patch 


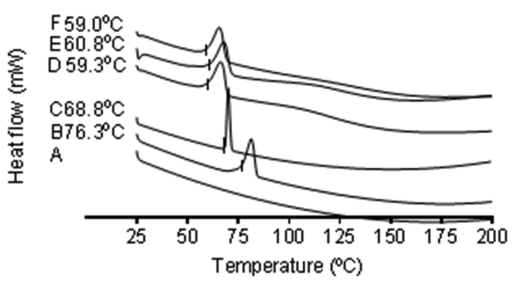

Figure 3. DSC heating profiles of (A) an empty pan, (B) lidocaine $\mathrm{HCl}$ powder, (C) lidocaine base powder, (D) PVP/RS100/PEO electrospun fibers, (E) PVP/RS100/PEO electrospun fibers containing lidocaine $\mathrm{HCl}$, and (F) PVP/RS100/PEO electrospun fibers containing lidocaine base. The onset melting temperatures of the upward-facing enthalpy peaks are given to the nearest $0.1{ }^{\circ} \mathrm{C}$. The image is representative of $n=2$ independent experiments.

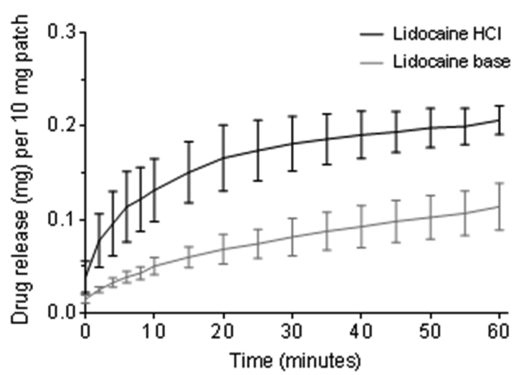

Figure 4. Accumulative release of lidocaine from the lidocaine $\mathrm{HCl}$ or lidocaine base-containing electrospun patches in PBS over $1 \mathrm{~h}$, shown as the drug released in $\mathrm{mg}$ per $10 \mathrm{mg}$ of the patch. Data presented is the mean $\pm \operatorname{SD}(n=4)$.

weights of $10.8 \pm 3.8$ and $11.20 \pm 2.7 \mathrm{mg}$ (mean $\pm \mathrm{SD})$. As there was variation in the patch weight and variation in drug loading, the drug release per $10 \mathrm{mg}$ of the patch was calculated. Lidocaine release from the lidocaine $\mathrm{HCl}$ patches increased rapidly to $0.16 \pm 0.04 \mathrm{mg}$ (mean $\pm \mathrm{SD}$ ) over the first $15 \mathrm{~min}$, then gradually at $0.21 \pm 0.02 \mathrm{mg}$ up to $60 \mathrm{~min}$. In contrast, the release of lidocaine base was slower, $0.06 \pm 0.01 \mathrm{mg}$ within 15 min, gradually increasing to $0.11 \pm 0.02 \mathrm{mg}$ after $1 \mathrm{~h}$ (Figure 4). Overall, the release of lidocaine from the lidocaine $\mathrm{HCl}$ patches was significantly quicker than that of lidocaine base from electrospun patches $(p<0.0001$; Mann-Whitney U test $^{31}$ ).

Permeation of Lidocaine $\mathrm{HCl}$ through ex vivo Porcine Buccal Mucosa. Dual-layer electrospun patches were used for buccal mucosal permeation studies ex vivo to ensure the unidirectional release of lidocaine directly into the mucosal tissue, thereby preventing release into the donor chamber. Accumulative permeation of lidocaine from patches containing between 0.20 and $0.35 \mathrm{mg}$ of lidocaine $\mathrm{HCl}$ per patch was linear over a period of $5 \mathrm{~h}$ (Figure 5), resulting in a flux of $0.268 \pm 0.009 \mu \mathrm{g} \mathrm{cm}^{-2} \mathrm{~min}^{-1}($ mean $\pm \mathrm{SD} ; n=6)$. In comparison, the application of $3 \%(\mathrm{w} / \mathrm{v})$ lidocaine $\mathrm{HCl}$ in the solution resulted in a flux of $4.092 \pm 0.062 \mu \mathrm{g} \mathrm{cm}^{-2} \mathrm{~min}^{-1}$ and $P_{\text {app }}$ value of $136.4 \pm 0.002\left(\times 10^{-3}\right) \mathrm{cm}^{-2} \mathrm{~min}^{-1}($ mean $\pm \mathrm{SD}$; $n=4$ ) (Figure S1). However, no lidocaine could be detected in the receptor buffer upon application of electrospun patches containing $0.25-0.39 \mathrm{mg}$ of lidocaine base per patch.

Spatial Distribution of Lidocaine $\mathrm{HCl}$ and Lidocaine Base Released from Electrospun Patches Applied to ex vivo Porcine Buccal Mucosa using MALDI-MSI. Timedependent drug penetration and localization using MALDIMSI was first investigated by exposure to a solution of lidocaine $\mathrm{HCl}$ (3 $\mathrm{mg} \mathrm{mL}^{-1}$, approximately $0.21 \mathrm{mg}$ applied) for

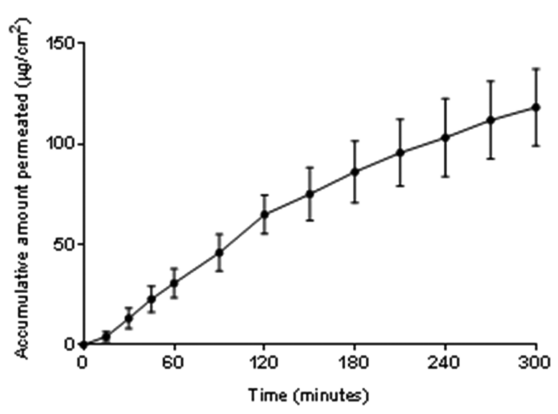

Figure 5. Accumulative permeation of lidocaine released from an electrospun patch through ex vivo porcine buccal mucosa. The electrospun patches contained between 0.20 and $0.35 \mathrm{mg}$ of lidocaine $\mathrm{HCl}$. Data are mean $\pm \mathrm{SD}(n=6)$.

up to $3 \mathrm{~h}$ (Figure 6). The control image with no lidocaine applied to the tissue shows that the lidocaine $\mathrm{m} / z$ signal is not present in the tissue. After $15 \mathrm{~min}$, lidocaine was located exclusively in the stratified epithelium, where it was evenly distributed and extending into the rete ridges. Drug penetration did not progress beyond the basement membrane. In contrast, after $1 \mathrm{~h}$, lidocaine was concentrated throughout the entire epithelium and was observed progressing into the lamina propria (Figure 6), and by $3 \mathrm{~h}$, lidocaine was found evenly distributed throughout the epithelium, lamina propria, and submucosa (Figure 6). The mass spectrum averaged over 100 pixels from this $3 \mathrm{~h}$ time point (Figure 6) is given in Figure S2, to show that the lidocaine signal was best detected in the protonated form at $\mathrm{m} / z 235.1807$ with a mass accuracy of 1.0 ppm.

Lidocaine released from the lidocaine $\mathrm{HCl}$ patches was visible in the stratified epithelium and extending into the lamina propria after just 15 min (Figure 7). After 1 and $3 \mathrm{~h}$, lidocaine was distributed throughout the epithelium and lamina propria (Figure 7). The mucosal distribution of lidocaine was more widespread and homogeneous when lidocaine $\mathrm{HCl}$ was applied as a solution compared to the electrospun patches (Figure 6). However, unlike administration of a solution in the oral cavity, the electrospun patches maintained local contact to the tissue where lidocaine was not depleted from the patch over a period of $3 \mathrm{~h}$ (Figure 7). Similar spatial distribution of lidocaine in tissue was observed from lidocaine base-containing patches applied to the ex vivo buccal tissue (Figure S3).

Lidocaine Released from Electrospun Patches Blocks Voltage-Gated Sodium Channels and Prevents Veratridine-Induced Calcium Responses in SH-SY5Y Neuroblastoma Cells. Veratridine, an alkaloid toxin found in Liliaceae plants, causes the persistent opening of voltage-gated sodium channels leading to cell depolarization and downstream intracellular calcium flux. ${ }^{32}$ Lidocaine has been shown to exert its biological action by blocking these voltage-gated sodium channels. ${ }^{33}$ To test whether the lidocaine released from electrospun patches remained functional, we examined its ability to block endogenously expressed voltage-gated sodium channels by examining intracellular calcium flux following cell stimulation with veratridine. Untreated SH-SY5Y cells displayed increased fluorescence associated with intracellular calcium flux when stimulated with veratridine $(17.9 \pm 2.8 \mathrm{RFU}$ over baseline; Figure 8A). Similar data were observed when SH-SY5Y cells were pretreated with the elutant from the placebo electrospun patches that contained no lidocaine (19.4 


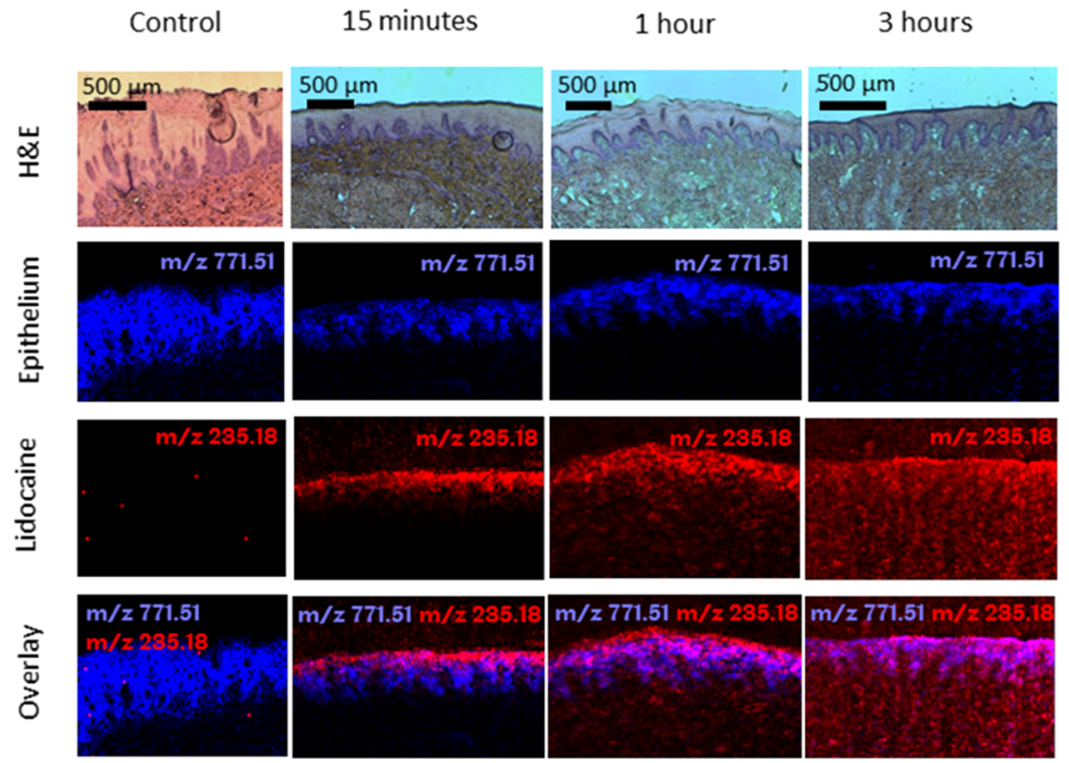

Figure 6. Hematoxylin and eosin (H\&E)-stained tissue sections and corresponding MALDI-MS images of porcine buccal mucosa as a control with no treatment and exposed to $0.3 \%(\mathrm{w} / \mathrm{v})$ lidocaine $\mathrm{HCl}$ solution $\left(\mathrm{m} / z 235.1805[\mathrm{M}+\mathrm{Na}]^{+}\right.$; red) after $15 \mathrm{~min}, 1$, and $3 \mathrm{~h}$. The epithelium $(\mathrm{blue})$ for each sample is shown using the epithelial marker lipid phosphatidylglycerol $(34: 1)\left(\mathrm{m} / z 771.5140[\mathrm{M}+\mathrm{Na}]^{+}\right)$.

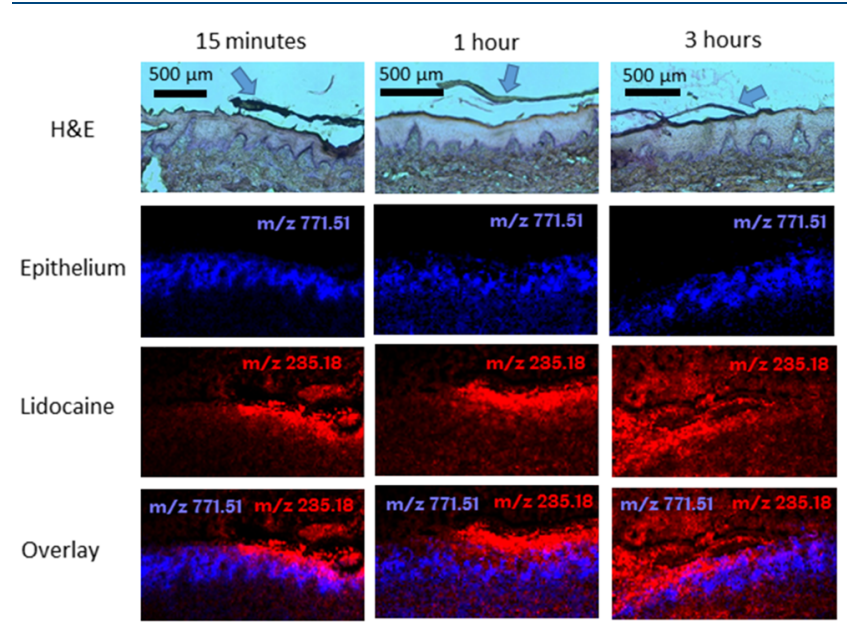

Figure 7. Hematoxylin and eosin (H\&E)-stained tissue sections and corresponding MALDI-MS images of porcine buccal mucosa exposed to dual-layer electrospun patches containing $3 \%(\mathrm{w} / \mathrm{v})$ lidocaine $\mathrm{HCl}$ $\left(\mathrm{m} / z 235.1805[\mathrm{M}+\mathrm{Na}]^{+}\right.$; red) after $15 \mathrm{~min}, 1$, and $3 \mathrm{~h}$. The epithelium (blue) for each sample is shown using the epithelial marker lipid phosphatidylglycerol $(34: 1)\left(\mathrm{m} / z\right.$ 771.5140 $\left.[\mathrm{M}+\mathrm{Na}]^{+}\right)$. The arrows in the H\&E images show the position of the electrospun patch.

\pm 3.0 RFU over baseline, Figure 8B). In contrast, intracellular calcium flux was significantly reduced $(11.3 \pm 4.8$ RFU over baseline; $p \leq 0.05$ ) compared to the placebo patch when cells were preincubated with the elutant from lidocaine $\mathrm{HCl}$ electrospun patches (Figure 8C,D), showing that the lidocaine released from the electrospun patches was functional and able to inhibit veratridine-mediated opening of voltage-gated sodium channels. The injection of DMSO alone as control did not induce a calcium flux fluorescence signal, whereas preincubation of cells with a solution of lidocaine $\mathrm{HCl}(10$ $\mathrm{mM}$ ) inhibited the veratridine-mediated calcium influx by $88 \%$ $(2.2 \pm 1.5$ RFU over baseline; $p \leq 0.05)$.
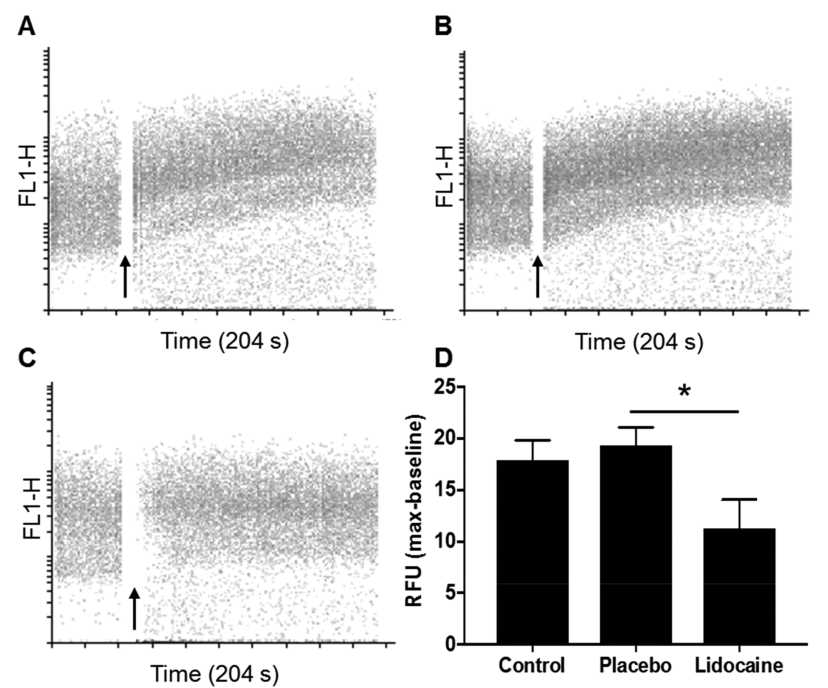

Figure 8. Calcium flux in SH-SYSY cells was determined overtime using flow cytometry. Baseline fluorescence was acquired for $40 \mathrm{~s}$ before injection of veratridine (black arrow) to induce a calcium influx. (A) Veratridine alone (control), (B) placebo patch elutant, and (C) lidocaine $\mathrm{HCl}$ patch elutant $(0.5 \mathrm{mM})$. Relative fluorescent units were determined by subtracting the median baseline from the maximum median fluorescence following stimulation with veratridine. Data presented is the mean $\pm \mathrm{SD}(n=3)$. Data were analyzed using a nonparametric one-way analysis of variance with a post-hoc KruskalWallis multiple comparison test, $* p \leq 0.05$.

\section{DISCUSSION}

Painful oral mucosal conditions and dental pain can greatly affect the quality of life. For dental treatments, injections are commonly used; however, this causes dental anxiety in many patients. A number of topical delivery methods of pain relief are available, ${ }^{34}$ although many are inadequate for targeting the affected local tissue and delivering a sustained drug release, where mucoadhesiveness and direct contact of the delivery vehicle to the local tissue are an underlying issue. We have 
previously developed a mucoadhesive electrospun oral drug delivery patch to resolve these issues and have used these patches to deliver the corticosteroid clobetasol-17-propionate to the mucosal tissue. ${ }^{11,12}$ The incorporation of lidocaine into electrospun polymers for transbuccal delivery has not previously been performed, although Palo et al. incorporated lidocaine $\mathrm{HCl}$ into an ink-jet-printed oral patch in a combined electrospun additive manufacturing process, ${ }^{35}$ thus not directly comparable to the patches used in this study. The data reported here confirms the ability of applying electrospinning to manufacture patches consisting of microscale polymer fibers loaded with lidocaine $\mathrm{HCl}$ and lidocaine base during fabrication. The incorporation of lidocaine $\mathrm{HCl}$ or base did not appear to influence fiber morphology, consistent with previous reports for unloaded and drug-loaded fibers. ${ }^{12,36}$ DSC analysis showed that both forms of lidocaine were amorphous when incorporated into electrospun fibers. Similar observations have been reported for many other drug compounds where the amorphous form has been shown to aid drug solubility and bioavailability. ${ }^{37-39}$

Following the manufacture of lidocaine-loaded patches, drug release studies were performed to compare release rates for lidocaine $\mathrm{HCl}$ or lidocaine base patches (Figure 4). Significantly greater lidocaine release was observed from lidocaine $\mathrm{HCl}$-containing patches. Around $80 \%$ of lidocaine was released from the electrospun fibers in $1 \mathrm{~h}$, which is similar to the previously reported release of lidocaine from poly-Llactic acid electrospun fibers containing lidocaine $\mathrm{HCl}^{40}$ Lidocaine base has been previously electrospun into $\mathrm{CMC} /$ PEO fibers, ${ }^{41}$ where release in the $1 \mathrm{~h}$ was close to $50 \%$, also agreeing with the findings in this study. One reason for the difference in release between the two drug compositions may be the surface $\mathrm{pH}$ microenvironment of the electrospun patches and the difference in acidity between the encapsulated lidocaine $\mathrm{HCl}$ and base. The acidic lidocaine $\mathrm{HCl}$ protonates and releases more quickly than that of lidocaine base, as shown in this study. The solubility of weakly basic drugs increases in lower $\mathrm{pH}$ environments, and hence acidity modifiers within the delivery vehicle may be used to increase their solubility. ${ }^{42}$

The lidocaine-loaded electrospun patches were subsequently investigated for their ability to deliver lidocaine to the porcine buccal mucosa. For this experiment, cryopreserved frozen porcine buccal mucosa was used, as previous studies have shown no change in the permeability barrier functions between fresh and frozen mucosal tissues. ${ }^{7,24}$ The permeation profile of lidocaine across porcine buccal mucosa released from a lidocaine $\mathrm{HCl}$-containing patch had a similar linear profile with a slight time lag as observed for the lidocaine permeating esophageal porcine tissue ${ }^{7}$ and rabbit ear skin ${ }^{43,44}$ after release from a lidocaine $\mathrm{HCl}$-containing polyvinyl alcohol film. The lidocaine apparent permeability coefficient in solution to cross porcine buccal epithelium has been previously reported as 17.0 $\pm 1.8\left(\times 10^{-6}\right) \mathrm{cm} \mathrm{s}^{-1}$, which is 7 times greater than shown in this study. ${ }^{17}$ However, here we have used a thicker section of mucosal tissue containing both epithelium and lamina propria compared to that study by Kokate et al. In the present study, lidocaine released from lidocaine base patches was not detected in the receptor chamber, suggesting that lidocaine release from the electrospun fibers was too slow, and/or the amount of permeated lidocaine through the tissue was too low to be detected by RP-HPLC. Lidocaine base has previously been reported to permeate the buccal tissue when released from a polymeric thin film, ${ }^{45}$ likely because the film contained approximately 100 times more lidocaine than in this study. Notably, the permeation of lidocaine observed in the study by Cavallari et al. was surprisingly quick as the authors were able to detect the drug in the receptor chamber after only $5 \mathrm{~min}$, and permeation was shown to be at a similar rate to its release rate in PBS despite the tissue thickness being reported to be $1.3 \mathrm{~cm}^{45}$

Although ex vivo permeation studies provide valuable information on the rate of permeation, these experiments do not provide information on the spatial localization of drugs within the tissue over time. MSI is a powerful tool for highresolution detection of substances in tissue sections, and a number of MSI techniques have been used to detect the presence of lidocaine, and metabolites thereof, in the skin. ${ }^{46-48}$ Here, we show for the first time the spatial distribution of lidocaine within porcine buccal mucosa following delivery from a mucoadhesive electrospun patch. Our data shows that lidocaine penetration into the oral tissue is most pronounced in the region of the patch placement, especially in the first 15 min, where lidocaine was mainly present in the stratified epithelium directly adjacent to the patch. For the lidocaine $\mathrm{HCl}$-containing patches, the lidocaine signal was most intense within the electrospun patch compared to the tissue, especially for $15 \mathrm{~min}$ and $1 \mathrm{~h}$. When associating this to the lidocaine permeation data where only $20 \mu \mathrm{g}$ of potentially up to $300 \mu \mathrm{g}$ of lidocaine contained in the patch had permeated through the mucosa after $1 \mathrm{~h}$, it is understandable that the signal in the patch is much stronger. Once released from the patch, lidocaine, being a lipophilic molecule, permeated through the epithelium and into the lamina propria over time that was consistent with the homogeneous time-dependent tissue distribution displayed by lidocaine $\mathrm{HCl}$ in the solution. The tissue penetration depth may also be dose-dependent, as has been shown previously following the application of lidocaine to the skin. ${ }^{47}$ MALDI-MSI also allowed us to review the localization of lidocaine released from the lidocaine basecontaining patches (Figure S3). Although lidocaine permeation was below the detection limit by HPLC, we found that lidocaine was localized throughout the epithelium and in the lamina propria after just $15 \mathrm{~min}$ exposure to the patch.

Although previous studies have shown the release of lidocaine from a number of fabricated films, none has shown that the drug retains its functional biological activity once released. Lidocaine exerts its anesthetic activity by blocking several voltage-gated sodium channels expressed by neuronal cells. ${ }^{33}$ Here, we show that lidocaine released from electrospun patches was able to interact with voltage-gated sodium channels expressed by SH-SY5Y neuroblastoma cells preventing their opening upon stimulation veratridine. ${ }^{32}$ These data give clear evidence that levels of lidocaine released from patches directly into tissue are therapeutically active, enabling blockage of oral mucosal neuronal cell depolarization.

\section{CONCLUSIONS}

Electrospun patches containing lidocaine HCL and lidocaine base were successfully manufactured by electrospinning, and the release of biologically active lidocaine from both types of the patch was demonstrated. Detailed investigations of diffusion through ex vivo porcine buccal mucosa suggested that lidocaine permeated into tissues following the release from a patch. This was confirmed by MALDI-MSI where, for the first time, the distribution of electrospun-delivered lidocaine within oral soft tissue was visualized. These data provide strong 
evidence that electrospun patches have potential as a delivery vehicle for lidocaine into buccal mucosa. Further clinical investigation is required to determine the feasibility of using this patch system as a topical dental anesthetic or as an analgesic for patients with painful oral mucosal disease conditions.

\section{ASSOCIATED CONTENT}

\section{S Supporting Information}

The Supporting Information is available free of charge on the ACS Publications website at DOI: 10.1021/acs.molpharmaceut.9b00535.

Accumulative permeation of lidocaine $\mathrm{HCl}$ through porcine buccal mucosa and additional mass spectrometry data (PDF)

\section{AUTHOR INFORMATION}

\section{Corresponding Authors}

*E-mail: christian.janfelt@sund.ku.dk (C.J.).

*E-mail: paul.hatton@sheffield.ac.uk (P.V.H.).

\section{ORCID $\odot$}

Craig Murdoch: 0000-0001-9724-122X

Sebastian Guy Spain: 0000-0001-7241-5713

Hanne Mørck Nielsen: 0000-0002-7285-9100

Christian Janfelt: 0000-0002-4626-3426

Paul V. Hatton: 0000-0001-5234-1104

\section{Author Contributions}

K.H.C. Conceptualization, Methodology, Investigation, Software, Writing: Original draft. C.M.: Conceptualization, Supervision, Visualization, Investigation, Writing: Reviewing and Editing. S.G.S.: Supervision, Methodology, Writing: Reviewing and Editing. A.M.H.: Validation, Writing: Reviewing and Editing. H.E.C.: Methodology, Investigation, Writing. M.B.S.: Methodology, Writing: Reviewing and Editing. H.M.N.: Methodology, Writing: Reviewing and Editing. C.J.: Methodology, Visualization, Writing: Reviewing and Editing, Supervision. P.V.H.: Supervision, Conceptualization, Data Curation, Writing. J.J.: Conceptualization, Methodology, Investigation, Supervision, Writing: Reviewing and Editing.

\section{Notes}

The authors declare the following competing financial interest(s): AFYX Therapeutics provided funding to provide partial support for the $\mathrm{PhD}$ studentship of Katharina Clitherow. Professor Paul V. Hatton is a member of the Scientific Advisory Board for AFYX Therapeutics.

\section{ACKNOWLEDGMENTS}

This work was funded by the U.K. EPSRC (EP/L016281/1) as a CASE Ph.D. studentship with the Centre for Doctoral Training in Polymers \& Soft Matter with sponsorship from AFYX Therapeutics as the industrial partner. Special thanks to Jens Hansen and Martin Santocildes-Romero at AFYX Therapeutics for acting as industrial advisors. Thank you to Rob Hanson and Yang Hwan Yun for technical advice in using the RP-HPLC.

\section{REFERENCES}

(1) Medicines Optimisation in Long-Term PainlGuidance and GuidelinesINICE. https://www.nice.org.uk/advice/ktt21/chapter/ Evidence-context (accessed Jan 14, 2019).
(2) Robertson, J. J. Managing Pharyngeal and Oral Mucosal Pain. Curr. Emerg. Hosp. Med. Rep. 2016, 4, 57-65.

(3) Rose-Ped, A. M.; Bellm, L.; Epstein, J. B.; Trotti, A.; Gwede, C.; Fuchs, H. Complications of Radiation Therapy for Head and Neck Cancers. Cancer Nurs. 2002, 25, 461-467.

(4) van Wijk, A. J.; Hoogstraten, J. Anxiety and Pain during Dental Injections. J. Dent. 2009, 37, 700-704.

(5) Sharmin, N.; Elias-Al-Mamun, M.; Islam, M. S.; Jalil, R. U. Preparation and Characterization of Lidocaine Double Layer Buccal Tablet Using Mucoadhesive Carbopol Polymers. Dhaka Univ. J. Pharm. Sci. 2011, 10, 29-34.

(6) Nafee, N. A.; Ismail, F. A.; Boraie, N. A.; Mortada, L. M. Mucoadhesive Delivery Systems. I. Evaluation of Mucoadhesive Polymers for Buccal Tablet Formulation. Drug Dev. Ind. Pharm. 2004, 30, 985-993.

(7) Padula, C.; Pozzetti, L.; Traversone, V.; Nicoli, S.; Santi, P. In Vitro Evaluation of Mucoadhesive Films for Gingival Administration of Lidocaine. AAPS PharmSciTech 2013, 14, 1279-1283.

(8) Morales, J. O.; McConville, J. T. Manufacture and Characterization of Mucoadhesive Buccal Films. Eur. J. Pharm. Biopharm. 2011, 77, 187-199.

(9) Fini, A.; Bergamante, V.; Ceschel, G. C. Mucoadhesive Gels Designed for the Controlled Release of Chlorhexidine in the Oral Cavity. Pharmaceutics 2011, 3, 665-679.

(10) Bågesund, M.; Tabrizi, P. Lidocaine 20\% Patch vs Lidocaine 5\% Gel for Topical Anaesthesia of Oral Mucosa. Int. J. Paediatr. Dent. 2008, 18, 452-460.

(11) Santocildes-Romero, M. E.; Hadley, L.; Clitherow, K. H.; Hansen, J.; Murdoch, C.; Colley, H. E.; Thornhill, M. H.; Hatton, P. V. Fabrication of Electrospun Mucoadhesive Membranes for Therapeutic Applications in Oral Medicine. ACS Appl. Mater. Interfaces 2017, 9, 11557-11567.

(12) Colley, H. E.; Said, Z.; Santocildes-Romero, M. E.; Baker, S. R.; D’Apice, K.; Hansen, J.; Madsen, L. S.; Thornhill, M. H.; Hatton, P. V.; Murdoch, C. Pre-Clinical Evaluation of Novel Mucoadhesive Bilayer Patches for Local Delivery of Clobetasol-17-Propionate to the Oral Mucosa. Biomaterials 2018, 178, 134-146.

(13) Sill, T. J.; von Recum, H. Electrospinning: Applications in Drug Delivery and Tissue Engineering. Biomaterials 2008, 29, 1989-2006.

(14) Samprasit, W.; Kaomongkolgit, R.; Sukma, M.; Rojanarata, T.; Ngawhirunpat, T.; Opanasopit, P. Mucoadhesive Electrospun Chitosan-Based Nanofibre Mats for Dental Caries Prevention. Carbohydr. Polym. 2015, 117, 933-940.

(15) Cantón, I.; Mckean, R.; Charnley, M.; Blackwood, K. A.; Fiorica, C.; Ryan, A. J.; MacNeil, S. Development of an IbuprofenReleasing Biodegradable PLA/PGA Electrospun Scaffold for Tissue Regeneration. Biotechnol. Bioeng. 2010, 105, 396-408.

(16) Gammaitoni, A. R.; Alvarez, N. A.; Galer, B. S. Safety and Tolerability of the Lidocaine Patch 5\%, a Targeted Peripheral Analgesic: A Review of the Literature. J. Clin. Pharmacol. 2003, 43, 111-117.

(17) Kokate, A.; Li, X.; Jasti, B. Effect of Drug Lipophilicity and Ionization on Permeability across the Buccal Mucosa: A Technical Note. AAPS PharmSciTech 2008, 9, 501-504.

(18) Hu, L.; Silva, S. M. C.; Damaj, B. B.; Martin, R.; MichniakKohn, B. B. Transdermal and Transbuccal Drug Delivery Systems: Enhancement Using Iontophoretic and Chemical Approaches. Int. J. Pharm. 2011, 421, 53-62.

(19) Collins, P.; Laffoon, J.; Squier, C. A. Comparative Study of Porcine Oral Epithelium. J. Dent. Res. 1981, 60, No. 543.

(20) Spengler, B. Mass Spectrometry Imaging of Biomolecular Information. Anal. Chem. 2015, 87, 64-82.

(21) Marxen, E.; Jacobsen, J.; Hyrup, B.; Janfelt, C. Permeability Barriers for Nicotine and Mannitol in Porcine Buccal Mucosa Studied by High-Resolution MALDI Mass Spectrometry Imaging. Mol. Pharm. 2018, 15, 519-526.

(22) Marxen, E.; Jin, L.; Jacobsen, J.; Janfelt, C.; Hyrup, B.; Nicolazzo, J. A. Effect of Permeation Enhancers on the Buccal 
Permeability of Nicotine: Ex Vivo Transport Studies Complemented by MALDI MS Imaging. Pharm. Res. 2018, 35, No. 70.

(23) Schneider, C. A.; Rasband, W. S.; Eliceiri, K. W. NIH Image to ImageJ: 25 Years of Image Analysis. Nat. Methods 2012, 9, 671-675.

(24) Marxen, E.; Axelsen, M. C.; Pedersen, A. M. L.; Jacobsen, J. Effect of Cryoprotectants for Maintaining Drug Permeability Barriers in Porcine Buccal Mucosa. Int. J. Pharm. 2016, 511, 599-605.

(25) Schramm, T.; Hester, A.; Klinkert, I.; Both, J. P.; Heeren, R. M. A.; Brunelle, A.; Laprévote, O.; Desbenoit, N.; Robbe, M. F.; Stoeckli, M.; et al. ImzML - A Common Data Format for the Flexible Exchange and Processing of Mass Spectrometry Imaging Data. J. Proteomics 2012, 75, 5106-5110.

(26) Robichaud, G.; Garrard, K. P.; Barry, J. A.; Muddiman, D. C. MSiReader: An Open-Source Interface to View and Analyze High Resolving Power MS Imaging Files on Matlab Platform. J. Am. Soc. Mass Spectrom. 2013, 24, 718-721.

(27) Janfelt, C.; Wellner, N.; Leger, P.-L.; Kokesch-Himmelreich, J.; Hansen, S. H.; Charriaut-Marlangue, C.; Hansen, H. S. Visualization by Mass Spectrometry of 2-Dimensional Changes in Rat Brain Lipids, Including N-Acylphosphatidylethanolamines, during Neonatal Brain Ischemia. FASEB J. 2012, 26, 2667-2673.

(28) Zamani, M.; Morshed, M.; Varshosaz, J.; Jannesari, M. Controlled Release of Metronidazole Benzoate from Poly $\varepsilon$ Caprolactone Electrospun Nanofibers for Periodontal Diseases. Eur. J. Pharm. Biopharm. 2010, 75, 179-185.

(29) Tonglairoum, P.; Ngawhirunpat, T.; Rojanarata, T.; Panomsuk, S.; Kaomongkolgit, R.; Opanasopit, P. Fabrication of Mucoadhesive Chitosan Coated Polyvinylpyrrolidone/Cyclodextrin/Clotrimazole Sandwich Patches for Oral Candidiasis. Carbohydr. Polym. 2015, 132, 173-179.

(30) Blagden, N.; de Matas, M.; Gavan, P. T.; York, P. Crystal Engineering of Active Pharmaceutical Ingredients to Improve Solubility and Dissolution Rates. Adv. Drug Delivery Rev. 2007, 59, 617-630.

(31) Cheung, Y. K.; Klotz, J. H. The Mann-Whitney Wilcoxon Distribution Using Linked Lists. Stat. Sin. 1997, 7, 805-813.

(32) Ulbricht, W. Effects of Veratridine on Sodium Currents and Fluxes. Rev. Physiol. Biochem. Pharmacol. 1998, 133, 1-54.

(33) Vetter, I.; Mozar, C. A.; Durek, T.; Wingerd, J. S.; Alewood, P. F.; Christie, M. J.; Lewis, R. J. Characterisation of Na v Types Endogenously Expressed in Human SH-SY5Y Neuroblastoma Cells. Biochem. Pharmacol. 2012, 83, 1562-1571.

(34) Lee, H.-S. Recent Advances in Topical Anesthesia. J. Dent. Anesth. Pain Med. 2016, 16, No. 237.

(35) Palo, M.; Kogermann, K.; Laidmäe, I.; Meos, A.; Preis, M.; Heinämäki, J.; Sandler, N. Development of Oromucosal Dosage Forms by Combining Electrospinning and Inkjet Printing. Mol. Pharm. 2017, 14, 808-820.

(36) Vrbata, P.; Berka, P.; Stránská, D.; Doležal, P.; Musilová, M.; Čižinská, L. Electrospun Drug Loaded Membranes for Sublingual Administration of Sumatriptan and Naproxen. Int. J. Pharm. 2013, 457, 168-176.

(37) Verreck, G.; Chun, I.; Rosenblatt, J.; Peeters, J.; Dijck, A.; Van Mensch, J.; Noppe, M.; Brewster, M. E. Incorporation of Drugs in an Amorphous State into Electrospun Nanofibers Composed of a WaterInsoluble, Nonbiodegradable Polymer. J. Controlled Release 2003, 92, 349-360.

(38) Yu, D. G.; Shen, X. X.; Branford-White, C.; White, K.; Zhu, L. M.; Annie Bligh, S. W. Oral Fast-Dissolving Drug Delivery Membranes Prepared from Electrospun Polyvinylpyrrolidone Ultrafine Fibers. Nanotechnology 2009, 20, No. 055104.

(39) Yu, D.-G.; Li, J.-J.; Williams, G. R.; Zhao, M. Electrospun Amorphous Solid Dispersions of Poorly Water-Soluble Drugs: A Review. J. Controlled Release 2018, 292, 91-110.

(40) Thakur, R. A.; Florek, C. A.; Kohn, J.; Michniak, B. B. Electrospun Nanofibrous Polymeric Scaffold with Targeted Drug Release Profiles for Potential Application as Wound Dressing. Int. J. Pharm. 2008, 364, 87-93.
(41) Maver, T.; Kurecic, M.; Smrke, D. M.; Kleinschek, K. S.; Maver, $\mathrm{U}$. Electrospun Nanofibrous $\mathrm{CMC} / \mathrm{PEO}$ as a Part of an Effective Pain-Relieving Wound Dressing. J. Sol-Gel Sci. Technol. 2016, 79, $475-486$.

(42) Dvořǎčková, K.; Doležel, P.; Mašková, E.; Muselík, J.; Kejdušová, M.; Vetchý, D. The Effect of Acid PH Modifiers on the Release Characteristics of Weakly Basic Drug from HydrophlilicLipophilic Matrices. AAPS PharmSciTech 2013, 14, 1341-1348.

(43) Padula, C.; Colombo, G.; Nicoli, S.; Catellani, P. L.; Massimo, G.; Santi, P. Bioadhesive Film for the Transdermal Delivery of Lidocaine: In Vitro and in Vivo Behavior. J. Controlled Release 2003, $88,277-285$

(44) Padula, C.; Nicoli, S.; Colombo, P.; Santi, P. Single-Layer Transdermal Film Containing Lidocaine: Modulation of Drug Release. Eur. J. Pharm. Biopharm. 2007, 66, 422-428.

(45) Cavallari, C.; Fini, A.; Ospitali, F. Mucoadhesive Multiparticulate Patch for the Intrabuccal Controlled Delivery of Lidocaine. Eur. J. Pharm. Biopharm. 2013, 83, 405-414.

(46) D’Alvise, J.; Mortensen, R.; Hansen, S. H.; Janfelt, C. Detection of Follicular Transport of Lidocaine and Metabolism in Adipose Tissue in Pig Ear Skin by DESI Mass Spectrometry Imaging. Anal. Bioanal. Chem. 2014, 406, 3735-3742.

(47) Kijima, S.; Todo, H.; Matsumoto, Y.; Masaki, R.; Kadhum, W. R; Sugibayashi, K. A Useful Technique Using Imaging Mass Spectrometry for Detecting the Skin Distribution of Topically Applied Lidocaine. J. Drug Delivery Sci. Technol. 2016, 33, 157-163.

(48) Eberlin, L. S.; Mulcahy, J. V.; Tzabazis, A.; Zhang, J.; Liu, H.; Logan, M. M.; Roberts, H. J.; Lee, G. K.; Yeomans, D. C.; Bois, J. Du.; et al. Visualizing Dermal Permeation of Sodium Channel Modulators by Mass Spectrometric Imaging. J. Am. Chem. Soc. 2014, 136, 64016405. 